\title{
The BRAF V600E Mutation is Not Present in All Cells of the Primary Melanoma and May Not Be Detected in All Metastatic Sites
}

\author{
Lars Alexander Schneider ${ }^{1, \S}$, Thomas F.E. Barth ${ }^{*}, 2$, , Margit Anna Huber ${ }^{1}$, Thomas Menzel ${ }^{3}$, \\ Karin Scharffetter-Kochanek ${ }^{1}$, Dietmar Thal ${ }^{2}$, Peter Möller ${ }^{2}$ and Ralf Marienfeld ${ }^{2}$ \\ ${ }^{I}$ Skin Cancer Center Ulm, University Department of Dermatology and Allergology Ulm, Germany \\ ${ }^{2}$ Department of Pathology and Neuropathology, University of Ulm, Germany \\ ${ }^{3}$ Dermatopathologie Friedrichshafen, D-88048 Friedrichshafen Bodensee, Germany
}

\begin{abstract}
The invention of targeted therapy for advanced stage melanoma has made it necessary to perform a mutation analysis of each melanoma case in order to detect the BRAF V600E mutation required for the prescription of selective BRAF inhibitors. In this new clinical context it is important to realize that the tumour seeds may come from different tumour clones and thus not all carry the mutation. We present an illustrative case that shows this phenomenon and discuss the consequences for clinical management in case of possible false negative mutation testing.
\end{abstract}

Keywords: Melanoma, BRAF V600E, mutation analysis, PCR, polyclonality.

\section{DEAR EDITOR,}

Recognition of the genetic profile of the BRAF V600E driver mutation [1-3] in malignant melanoma has led to the development of inhibitors blocking specifically mutated $B R A F$ [2, 4, 5]. This innovation renders metastatic melanoma a disease treatable with a selective targeted therapy in the palliative situation. Worldwide we face about 100,000 new melanoma patients per year in the caucasian population of Europe, North America, New Zealand, and Australia [5]. Genetic studies estimate that about $50 \%$ of these patients carry a V600E BRAF mutation [6]. However, in the first instance there is a need for a mutational analysis of excised tumour tissue prior to starting therapy since only patients with the BRAFV600E mutation benefit from this targeted therapy. The mutation analysis can be done from archived formalin-fixed and paraffin-embedded tumour material. The question "mutated or not?" has to be answered fairly quickly. Time is short and thus one might take the next best available tumour tissue specimen for a "pars pro toto" analysis assuming it is sufficient to determine the $B R A F$ V600E status. However, such an analysis on the basis of a single specimen may lead to a false negative result. A first report from Lin and colleagues from 2011 stated that melanoma is microheterogenous, and mutant alleles are selected during progression [7]. Others found this

*Address correspondence to this author at the Institute of Pathology, Ulm University, Ulm, Albert-Einstein- Allee 11, D89081 Ulm, University of Ulm, D-89081 Ulm, Germany; Tel: 0049 731500-56340; Fax: $0049731500-$ 56380; E-mail: thomas.barth@uniklinik-ulm.de

${ }^{\S}$ These authors contributed equally. heteroclonality on a single cell level in circulating blood melanoma cells [8]. One of our patients taught us that melanoma metastases are clonal evolutions and, thus, $B R A F$ $\mathrm{V} 600 \mathrm{E}$ analysis should be performed from different sites, if a wildtype status is detected in one probe.

\section{CASE REPORT (FIG. 1)}

A 38-year-old female patient was diagnosed having malignant melanoma of the right thumb close to the nail in September 2005. After complete resection and sentinel biopsy plus initial clinical staging, the initial status was pT3a pN1a cM0 AJCC stage IIIa. After adjuvant high dose Interferon therapy, which was terminated after 10 weeks because of severe fatigue grade IV according to the National Cancer Institute Common Toxicity Criteria (NCI-CTC), the patient was relapse free. Four years later in 2009 she progressed to stage IV disease with lung metastases and lymph node metastases in the left axilla. Both were resected. In 2010, the patient then developed a solitary occipital brain metastasis. Again the seed was resected. The first mutation analysis 2009 from microdissected material (tumour load $>90 \%$ ) of the axillary lymph nodes showed no mutation in axon $9,11,13$ and 17 of $C-K I T$ and also no mutation in codon 61 of $N-R A S$. Furthermore, the BRAF status as determined by pyrosequencing was wildtype. In 2010, the analysis was performed on material from the brain metastasis. In this material, the $B R A F$ V600E mutation was detected (approx. $31 \%$ mutated $B R A F$ allele). The analysis for $c-K I T$ was proving a wild-type sequence as before. We then proceeded and analysed microdissected material from the primary tumour from 2005; herein we found the $B R A F$ V600E mutation in about $40 \%$ of the analysed $B R A F$ alleles. We then tested the lung metastases from 2009; the $B R A F$ 
A

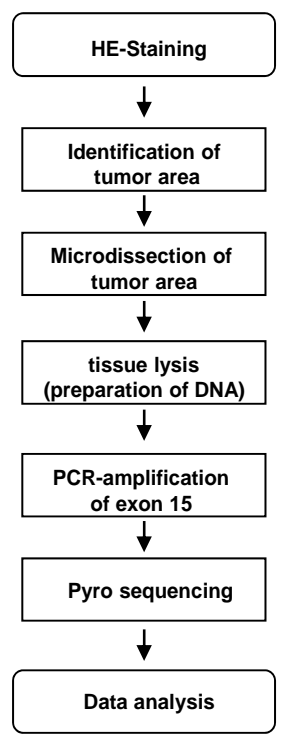

B

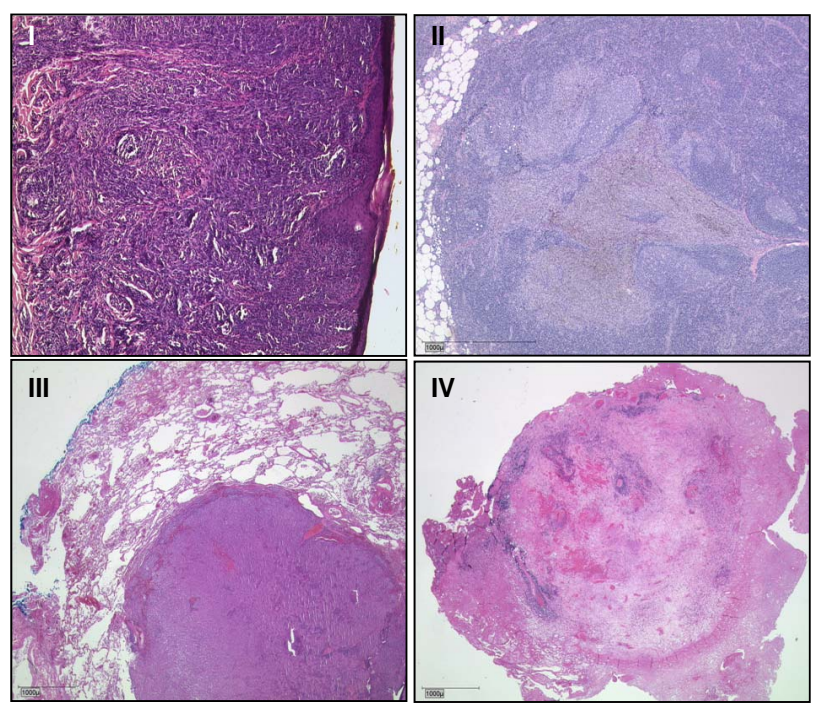

C
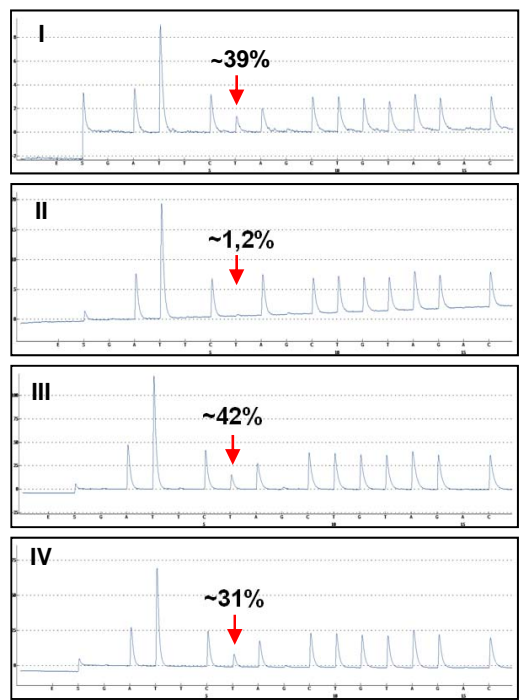

Fig. (1). A: Flow chart of the BRAF V600E mutational analysis leading to enriched tumor DNA for analysis. The mutational status at position 600 of the BRAF cDNA was assessed by reverse pyrosequencing. In brief, the tumor containing areal of the FFPE tissue was isolated by microdissection and the extracted genomic DNA was used to amplify Exon 15 by PCR (for: 5'-biotin-tgcttgctctgataggaaaatg-3', rev: 5'-agcatctcagggccaaaaat-3'). Subsequently, the area encompassing codon 600 of the BRAF cDNA was analysed by revers pyrosequencing (PyroMark Q24, protocol according to manufacturer, sequencing primer: 5'-GACCCACTCCATCGAG-3'). (B) Histomorphology of the primary melanoma (I), the metastases of the lymph node, lung and brain are shown in II-IV. The respective reverse pyrograms of these lesions are shown in C. (C) Quantification of mutated alleles was done by dividing the values for dispersions 6 ( $\mathrm{T}$, mut) and 7 (A, WT) by the sum of both values as described by Lennerz et al. [9].

$\mathrm{V} 600 \mathrm{E}$ mutation was found in the lung metastasis (in approx. $42 \%$ of analysed alleles).

We conclude that the axillary seed was a clonal seed from a wildtype fraction within the primary melanoma. Without a repetitive analysis from sequentially excised metastases from different sites and the primary tumour, the $B R A F$ V600E mutation in this case would have been missed, same as described only in one other case report so far [10]. Our patient was then treated with a selective BRAF inhibitor. Clinically after 9 months on this therapy she remains progression free at present such as expected under this kind of treatment [11].

Conclusions from this case are:

1. Melanoma spreads in subclones and not all of these may carry a $B R A F$ V600E mutation even though it may be present in other subclones of the primary lesion $[8,10]$.

2. The clinical consequence must be therefore to repeat testing in patients with a single wildtype testing result either from the primary tumour or from another metastastic sites [9].

3. BRAF testing is also worthwhile being considered when the melanoma lies on distant extremities as these tumours can have the mutation as well although typical for such acrolentiginous sites are $c-K I T$ mutations.

\section{CONFLICT OF INTEREST}

M.A. Huber and L.A. Schneider received travel grants from Roche, MSD and Bristol Myers Squibb.

L. A. Schneider and M. A. Huber served in advisory boards for Roche and Bristol Myers Squibb.

\section{ACKNOWLEDGEMENTS}

Declared none.

\section{REFERENCES}

[1] Curtin JA, Fridlyand J, Kageshita T, et al. Distinct sets of genetic alterations in melanoma. N Engl J Med 2005; 353(20): 2135-47.

[2] Dankort D, Curley DP, Cartlidge RA, et al. Braf(V600E) cooperates with Pten loss to induce metastatic melanoma. Nat Genet 2009; 41(5): 544-52.

[3] Fecher LA, Amaravadi RK, Flaherty KT. The MAPK pathway in melanoma. Curr Opin Oncol 2008; 20(2): 183-9.

[4] Flaherty KT, Hodi FS, Bastian BC. Mutation-driven drug development in melanoma. Curr Opin Oncol 2012; 22(3): 178-83.

[5] Houben R, Becker JC, Kappel A, et al. Constitutive activation of the Ras-Raf signaling pathway in metastatic melanoma is associated with poor prognosis. J Carcinog 2004; 3(1): 6 .

[6] Wan PT, Garnett MJ, Roe SM, et al. Mechanism of activation of the RAF-ERK signaling pathway by oncogenic mutations of BRAF. Cell 2004; 116(6): 855-67. 
[7] Lin J, Goto Y, Murata H, et al. Polyclonality of BRAF mutations in primary melanoma and the selection of mutant alleles during progression. Br J Cancer 2011; 104(3): 464-8.

[8] Sakaizawa K, Goto Y, Kiniwa Y, et al. Mutation analysis of BRAF and KIT in circulating melanoma cells at the single cell level. Br J Cancer 2012; 106(5): 939-46.

[9] Lennerz JK, Klaus BM, Marienfeld RB, Moller P. Pyrosequencing of BRAF V600E in routine samples of hairy cell leukaemia identifies CD5+ variant hairy cell leukaemia that lacks V600E. Br J Haematol 2012; 157(2): 267-9.

[10] Richtig E, Schrama D, Ugurel S, et al. BRAF mutation analysis of only one metastatic lesion can restrict the treatment of melanoma: a case report. Br J Dermatol 2012; DOI: 10.1111/j.1365-2133.2012. 11121.x.

[11] Hafner C. Treatment of metastatic melanoma with BRAF inhibitors. Hautarzt 2012; 62(9): 696-8.

(C) Schneider et al.; Licensee Bentham Open.

This is an open access article licensed under the terms of the Creative Commons Attribution Non-Commercial License (http://creativecommons.org/licenses/by$\mathrm{nc} / 3.0 /$ ) which permits unrestricted, non-commercial use, distribution and reproduction in any medium, provided the work is properly cited. 